\title{
ROTURA UTERINA EN PACIENTES SIN CICATRIZ UTERINA*
}

\author{
Drs. Cristián Belmar J., José A. Poblete L., Fernando Abarzúa C., Enrique Oyarzún E. \\ Departamento de Obstetricia y Ginecología, Pontificia Universidad Católica de Chile
}

\section{RESUMEN}

La rotura uterina se presenta habitualmente en pacientes con cicatriz de cesáreas previas, siendo la rotura de un útero sin cicatriz un fenómeno infrecuente, pero de graves consecuencias para la madre y el feto.

Presentamos nuestra experiencia reciente, detallando el manejo y resultados maternos y fetales.

\section{PALABRAS CLAVES: Rotura uterina sin cicatriz previa}

\section{SUMMARY}

Uterine ruptures is more common in women with previous cesarean deliveries, ruptures in unscarred uterus are unfrequent, but with serious consequences for the mother and fetus.

This report shows our recent experience, and describes treatment, and maternal and fetal outcomes.

KEY WORDS: Uterine rupture, unscarred uterus

\section{INTRODUCCION}

En 1881, se publica la primera serie de roturas uterinas, los 100 casos reportados ocurridos en úteros intactos (1), ya que en aquella época no era factible realizar una cesárea sin incrementar significativamente el riesgo de muerte materna. Con el transcurso del tiempo las distintas series están constituidas, cada vez por un mayor número de casos de rotura uterina en pacientes con cicatriz de cesárea. En la actualidad, este antecedente es el principal factor de riesgo para presentar una rotura uterina. Más aún, existe controversia respecto del cual sería la mejor opción de manejo para pacientes con cicatrices uterinas (2-5).

La posibilidad de rotura de un útero sin cicatriz es muy baja, $y$, a diferencia de las roturas en úteros con cicatriz, estos casos parecen ir en disminución $(6,7)$. A la fecha, y desde el reporte de Ames (1), hay no más de 500 casos en la literatura. Se han descrito, sin embargo, algunos factores de riesgo (8), entre los que se mencionan la multiparidad, macrosomía, uso de ocitocina, versiones fetales y anomalías uterinas. A pesar de esto, la mayoría de los casos se presentan de forma imprevista, y con complicaciones maternas y fetales variables según las distintas series (9).

El presente reporte comunica una serie de seis casos manejados en nuestro servicio, respecto de los cuales analizamos factores de riesgo, forma de presentación y resultados maternos y perinatales.

\section{PACIENTES Y METODOS}

Se revisaron los registros de partos de nuestro servicio en el período comprendido entre enero de 1995 y diciembre de 2001. Se incluyeron todos los casos con rotura de útero en todo su espesor y que

*Trabajo leído en la sesión del 2 de julio de 2002 de la Sociedad Chilena de Obstetricia y Ginecología. 
requirieron cirugía como parte de su tratamiento. Se obtuvo información detallada de las respectivas fichas clínicas.

\section{RESULTADOS}

En el período estudiado hubo un total de 19.158 partos en la maternidad del Hospital Clínico de la Pontificia Universidad Católica de Chile. Seis roturas de úteros en pacientes sin cicatriz se identificaron en dicho período. El porcentaje promedio de pacientes con cicatriz uterina fue de $16,2 \%$, lo cual da una incidencia de rotura en úteros sin cicatriz de 1 cada 3.737 partos.

Las características generales de las pacientes se ilustran en la Tabla I.

Cinco de los seis casos se presentaron durante el trabajo de parto a término, y un caso se presentó en una embarazada de 28 semanas de gestación. Este último caso correspondió a una paciente con dos partos vaginales previos y un aborto de segundo trimestre, quién cursando embarazo de $28 \mathrm{se}$ manas, presentó un cuadro de abdomen agudo y sufrimiento fetal; fue sometida a operación cesárea de urgencia obteniéndose un reciente nacido de 1.020 gramos con Apgar de 1 y 5 al minuto y cinco minutos respectivamente, y cuyos gases de cordón fueron $\mathrm{pH} 6,63, \mathrm{PaO}_{2} 16, \mathrm{PaCO}_{2}, 127, \mathrm{BE}-19,1$, quien falleció a los 6 días de vida postnatal. El útero mostró una rotura a nivel fúndico que se suturó sin inconvenientes.

De las pacientes con rotura uterina en trabajo de parto, éste se inició espontáneamente en 2 de ellas, mientras que en las otras 3 fue inducido; en dos casos por síndrome hipertensivo del embarazo, y en el otro por diabetes gestacional. La duración del trabajo de parto fue de 4,6 horas en promedio en aquellas en que se inició espontáneamente y de 9,7 horas en quienes fue inducido. Las cinco pacientes recibieron anestesia peridural y cuatro de

\section{Tabla}

\section{CARACTERISTICAS GENERALES DE LAS PACIENTES QUE PRESENTARON ROTURA UTERINA SIN CICATRIZ PREVIA}

\begin{tabular}{lll}
\hline & Promedio & Rango \\
\hline Edad & 33 años & $26-40$ años \\
Paridad & 1,66 partos & $1-3$ partos \\
$\begin{array}{l}\text { Edad gestacional } \\
\text { al parto }\end{array}$ & 37,08 semanas & $28-39,5$ semanas \\
$\begin{array}{l}\text { Peso recién } \\
\text { nacido }\end{array}$ & 3.720 gramos & $1.020-4.170$ gramos \\
\hline
\end{tabular}

ellas recibieron ocitocina; una recibió misoprostol. Las cinco pacientes tuvieron un parto vaginal, sólo en una de ellas fue asistido con fórceps bajo por bradicardia fetal, y ninguna presentó alteración del alumbramiento que hiciese sospechar una adhesividad placentaria anormal.

En relación con el diagnóstico, a excepción de la paciente sin trabajo de parto, en las demás pacientes se hizo el diagnóstico de rotura después del expulsivo. En dos casos el diagnóstico se hizo por la salida de epiplón a través de la vagina luego del parto; en los tres restantes por metrorragia postparto que en un caso obligó a laparotomía inmediata por sangrado incoercible, y en los otros dos casos a laparotomía exploradora, 3 y 5 horas postparto, con diagnóstico de inercia refractaria a tratamiento médico. En los cinco casos el hallazgo fe una rotura segmentaria con compromiso de alguna de las caras laterales del útero.

La mortalidad perinatal en nuestra serie fue de $16,6 \%(1 / 6)$, dos de los recién nacidos requirieron hospitalización y cinco de seis fueron dados de alta con su madre. En tres de los seis casos se presentó bradicardia fetal, en dos de estos durante el período expulsivo. Tres de los seis casos tuvieron apgar $<$ a 7 al minuto, y no hubo casos de apgar < a 3 a los 5 minutos. Sólo uno de los recién nacidos pesó más de 4.000 gramos al nacer.

No hubo muertes maternas en nuestra serie; tres pacientes requirieron histerectomía y en las otras tres se logró reparar el útero. La complicación común fue hemorragia, requiriendo cuatro de las seis pacientes transfusión de hemoderivados. El promedio de unidades de glóbulos rojos utilizados fue de siete en esas pacientes. No hubo complicaciones infecciosas ni lesiones de otros órganos. El promedio de días de hospitalización fue de 5,2.

\section{DISCUSION}

Tradicionalmente los mayores factores predisponentes para la rotura uterina han sido la gran multiparidad y el trauma obstétrico, a expensas de trabajos de partos prolongados, fetos macrosómicos, distocias de presentación, versiones externas e internas, partos en podálica y partos instrumentalizados $(7,8,10,11)$. Actualmente la macrosomía y las distocias de presentación se diagnostican con más frecuencia con ultrasonido, y los partos prolongados e instrumentalizados han sido desplazados por la cesárea, lo que explica que casos como los mostrados en este reporte sean cada vez menos frecuentes. La incidencia aquí reportada, concuerda, por otra parte, con lo descrito en la literatura $(6,7)$. 
En nuestra casústica, la mayoría de las pacientes presentó alguno de los factores de riesgo descritos por Sweeten y cols. (8); sin embargo, destaca en nuestros casos que en un $50 \%$ de ellos se presentó la complicación en partos inducidos, uno de ellos con uso de misoprostol ${ }^{\circledR}$. El uso de este medicamento se ha asociado a rotura uterina $(12,13)$.

Respecto al diagnóstico, se debe considerar que los signos clásicos de rotura se presentan tan sólo en un tercio de los casos (14). Debe considerarse además, que un sangrado postparto prolongado debe hacer sospechar esta complicación, como ocurrió en dos de nuestros casos. Lo anterior habría recomendable una exploración manual del útero rutinaria, ya que por una parte, esa maniobra no se asocia a un aumento de morbilidad febril significativa; y, por otra, Beacham y cols. (15) reportaron que la falla en explorar el útero fue responsable de la mitad de las muertes maternas en su casuística. En nuestra propia serie, de hecho, ésta conducta nos habría permitido tener una resolución quirúrgica más precoz, y probablemente evitar el uso de hemoderivados en dos casos.

Coincidentemente con lo reportado, todas nuestras pacientes tuvieron roturas que comprometieron el segmento uterino; y en el $50 \%$ de ellas, se realizó histerectomía, lo cual es menor a lo comunicado (16). Lo anterior, se explica, probablemente, por la baja paridad promedio de nuestras pacientes, lo que motivo una conducta más conservadora.

Existe preocupación en relación con el riesgo de recurrencia de rotura que se ha reportado entre 4 y $20 \%(17,18)$. Nuestras pacientes no han tenido nuevos embarazos, en cuyo caso la recomendación sería el practicar una operación cesárea electiva antes del inicio del trabajo de parto.

Los resultados perinatales han mostrado (19) tasas de mortalidad que fluctúan entre 5 y $40 \%$, en general debido a asfixia perinatal, la que fue la causa de muerte en el único caso de nuestra serie.

La mortalidad materna reportada $(19,20)$ ha sido bajo $5 \%$; sin embargo, la necesidad de transfundir hemoderivados, así como las complicaciones quirúrgicas e infecciosas son frecuentes. Evidencia de lo anterior, en nuestra casuística, fue la prolongación de la estadía de las pacientes, y la necesidad de transfusiones.

Debemos finalmente hacer notar que de las pacientes presentadas ninguna presentaba factores de alto riesgo perinatal; lo cual reafirma la conveniencia de la resolución del parto en lugares que cuenten con la infraestructura humana y técnica adecuada para enfrentar estas infrecuentes, pero graves complicaciones.

\section{BIBLIOGRAFIA}

1. Ames RPM: Rupture of the uterus. Am J Obstet Gynecol 1881; 14: 361-95.

2. Rageth JC, Juzi C, Grossenbacher H: Delivery after previous cesarean: a risk evaluation. Obstet Gynecol 1999; 93: 332-7.

3. Gregory KD, Korst LM, Cane P, Platt LD, Kahn K: Vaginal birth after cesarean and uterine rupture rates in California. Obstet Gynecol 1999; 94: 985-9.

4. McMahon MJ, Luther ER, Bowes WA Jr, Olshan AE: Comparison of a trial of labor with an elective second cesarean section. N Engl J Med 1996; 335: 689-95.

5. Lyndon M, Holt V, Easterling T, Martin D: Risk of uterine rupture during labor among women with a prior cesarean delivery. N Engl J Med 2001; 345: 3-8.

6. Miller D, Goodwin M, Gherman R, Paul R: Intrapartum rupture of the unscarred uterus. Obstet Gynecol 1997; 89: 671-3.

7. Eden R, Parker R, Gall S: Rupture of the pregnant uterus: A 53 year review. Obstet Gynecol 1986; 68(5): 671-4.

8. Sweeten K, Graves W, Athanassiou A: Spontaneous rupture of the unscarred uterus. Am J Obstet Gynecol 1995; 172: 1851-6.

9. Leung AS, Leung EK, Paul RH: Uterine rupture after previous cesarean delivery: maternal and fetal consequences. Am J Obstet Gynecol 1993; 169: 689-94.

10. Ferguson RK, Reid DE: Rupture of the uterus: A twenty-year report from the Boston Lying in Hospital. Am J Obstet Gynecol 1958; 76: 172-80.

11. Ware $H H$, Jarrett $A Q$, Reda $F A$ : Rupture of the gravid uterus: Report of 40 cases. Am J Obstet Gynecol 1958; 76: 181-7.

12. Bennett $B$ : Uterine rupture during induction of labor at term with intravaginal misoprostol. Obstet Gynecol 1997; 89: 832-3.

13. Khabbaz AY, Usta IM, El-Haij MI, Abu-Musa A, Seuod $M$, Nassar AH: Rupture of an unscarred uterus with misoprostol induction: case report and review of the literature. J Matern Fetal Med 2001; 10(2): 141-5.

14. Schrinsky DC, Benson RC: Rupture of the pregnant uterus. Obstet Gynecol Surv 1978; 33: 217.

15. Beacham WD, Beacham DW, Webster HD et al: Rupture of the uterus at New Orleans Charity Hospital. Am J Obstet Gynecol 1970; 106: 1083.

16. Saulding L, Gallup D: Current concepts of management of the rupture of the gravid uterus. Obstet Gynecol 1979; 54(4): 437-41.

17. Sheth SS: Results of treatment of rupture of the uterus by suturing. J Obstet Gynecol $\mathrm{Br}$ Common 1968; 75: 55-8.

18. Agüero $\mathrm{O}$, Kizer S: Obstetric prognosis of the repair of the uterines rupture. Surg Gynecol Obstet 1968; 127: 528-30.

19. Yap OW, Kim ES, Laros RK: Maternal and neonatal outcomes after uterine rupture in labor. Am $\mathrm{J}$ Obstet Gynecol 2001; 184(7): 1571-81.

20. Yazigi CA, Heinne RM: Muerte materna causada por rotura uterina espontánea en una primípara. Rev Chil Obstet Ginecol 1991; 56(4): 291-2. 


\section{DISCUSION}

Dr. Atala: Destaca la influencia del acretismo placentario y de la maniobra de Kristeller en este tipo de roturas.

Dr. Silva: Señala la importancia de la atención profesional del parto, que incluye el manejo adecuado de complicaciones como la rotura uterina.

Dr. Herrera: Clasifica las roturas uterina asociada a un trabajo de parto espontáneo o con intervención médica por maniobras.

El examen manual intrauterino es importante en la evaluación de la metrorragia temprana del puerperio. Agrega que los problemas legales se producen por la concepción social razonable de que el parto es un proceso fisiológico normal, siempre en mujeres sanas y libres de complicaciones.
Dr. Ramírez: Comenta sobre la posibilidad de un tratamiento conservador en la rotura uterina (o dehiscencia) cubierta por peritoneo. También pregunta sobre el resultado de anatomía patológica para descartar la presencia de acretismo placentario.

Dr. Guzmán: Pregunta más detalles sobre el caso inhabitual de la paciente con rotura uterina y embarazo de 28 semanas.

Dr. Belmar: Se realizó estudio de anatomía patológica en 3 pacientes, no se encontró acretismo placentario. No se tiene información sobre maniobras de Kristeller en los casos presentados. El manejo conservador de la rotura uterina incompleta a nivel de la cicatriz pequeña y cubierta por peritoneo es factible. En el manejo de estas pacientes es muy importante la experiencia y preparación del equipo médico a cargo. 